\title{
Nonlinear Magneto-Optical Rotation via Alignment-to-Orientation Conversion
}

\author{
D. Budker ${ }^{1,2, *}$, D. F. Kimball ${ }^{1}$, S. M. Rochester ${ }^{1}$, and V. V. Yashchuk $k^{1,3}$ \\ ${ }^{1}$ Department of Physics, University of California at Berkeley, Berkeley, California 94720-7300 \\ ${ }^{2}$ Nuclear Science Division, Lawrence Berkeley National Laboratory, Berkeley, California 94720 \\ ${ }^{3}$ B.P.Konstantinov Petersburg Nuclear Physics Institute, Gatchina, Russia 188350
}

(March 9, 2000)

\section{Report LBNL-45259}

Nonlinear magneto-optical rotation (NMOR) is investigated at high light powers where the rotation is significantly modified by AC Stark shifts. These shifts are shown to change the overall sign of rotation for closed $F \rightarrow F+1$ transitions as light power is increased. The effect is demonstrated by measurements in rubidium and density matrix calculations. The results are important for applications of nonlinear optical rotation such as sensitive magnetometry.

PACS. $32.60+\mathrm{i}, 42.50 . \mathrm{Gy}, 32.80 . \mathrm{Bx}, 42.50 . \mathrm{Hz}$

Nonlinear magneto-optical rotation (NMOR) arises when sufficiently intense light interacts with atoms in the presence of a magnetic field $[1,2]$. Recently, two approaches to NMOR-based magnetometry, one involving ultranarrow $(1 \mathrm{~Hz})$ resonances [3,4], and the other using high density atomic samples [5], have been investigated. The optimum sensitivity in both approaches is achieved for light powers where the number of optical pumping cycles during the relaxation time of ground state coherences exceeds unity. Here we show that under such conditions the dominant physical mechanism causing NMOR can be quite different from that in the low light power regime (well described by a perturbative model [6]). In particular, we show that alignment-to-orientation conversion [7-12] due to the combined action of the magnetic field and light electric field is the primary mechanism responsible for NMOR at the light frequencies and intensities where the highest sensitivity to magnetic fields is achieved [4].

Here we consider nonlinear magneto-optic effects related to the evolution of atomic polarization moments (the coherence effects) in the Faraday geometry, where the magnetic field is oriented along the direction of light propagation. For low light intensities, the coherence effects are well described in terms of a three stage process [6,3]: (1) atoms are optically pumped into an aligned state, causing the atomic vapor to acquire linear dichroism, (2) the atomic alignment precesses in the magnetic field, rotating the axis of dichroism, and (3) the light polarization is rotated by interaction with the dichroic atomic medium, since the alignment is no longer along the direction of light polarization. This model predicts that for closed transitions, the sign of NMOR for $F \rightarrow F-1, F$ transitions should be opposite to the sign of optical rotation for $F \rightarrow F+1$ transitions [13] (where $F$ is the total angular momentum in the ground state), as has been observed for relatively low light intensities [6,3]. However, as light power is increased, we have observed that NMOR for closed $F \rightarrow F+1$ transitions changes sign - indicating that a different physical mechanism becomes dominant.

The setup for the present measurements is shown in Fig. 1. In Fig. 2, NMOR spectra for the rubidium D2 line $(780 \mathrm{~nm})$ at a fixed magnetic field at two different light powers are compared to density matrix calculations [14]. A density matrix is written for each ground state hyperfine level containing the ground state and all excited states accessible via optical transitions. The effects of repopulation pumping are included in the calculations. Note that the sign of rotation for the ${ }^{87} \mathrm{Rb} F=2 \rightarrow 3$ and ${ }^{85} \mathrm{Rb} F=3 \rightarrow 4$ components reverse at the higher light power.

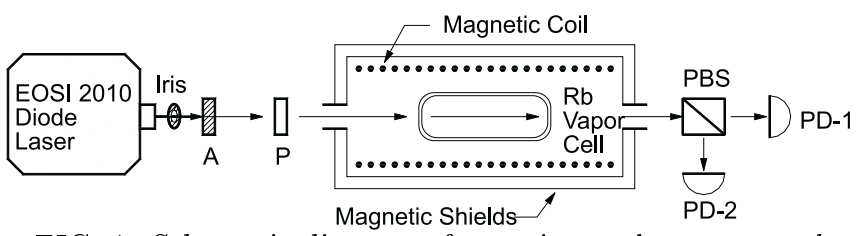

FIG. 1. Schematic diagram of experimental setup, see also Ref. [2]. The laser is an external cavity diode laser (EOSI 2010). The uncoated cylindrical glass vapor cell contains a natural isotopic mixture of Rb. A - attenuator, $\mathrm{P}$ - linear polarizer, PBS - polarizing beamsplitter used to measure polarization rotation, PD-1,2 - photodiodes.

In order to illustrate the mechanisms responsible for this effect, we consider the simpler cases of closed, spectrally isolated $1 \rightarrow 0$ and $1 \rightarrow 2$ transitions with separated pump, precession, and probe fields (Fig. 3). The pump light frequency is held fixed on resonance and the power corresponds to the optical pumping saturation parameter $\kappa=d^{2} E_{0}^{2} /\left(\gamma_{0} \gamma_{t}\right) \approx 1$, where $d$ is the transition dipole moment, $E_{0}$ is the amplitude of the light electric field, and $\gamma_{t}$ is the transit rate of the atoms, chosen to be the same for each region. The probe light power corresponds to $\kappa \ll 1$. In the precession region there is a magnetic field in the $\hat{z}$ direction of magnitude $B_{z} \ll \gamma_{t} /\left(2 g_{F} \mu\right)$ where $g_{F}$ is the ground state Landé factor and $\mu$ is the Bohr magneton. To simulate the effect of AC Stark shifts on precession in the experiment, a DC electric field is applied in the $\hat{x}$ direction. 


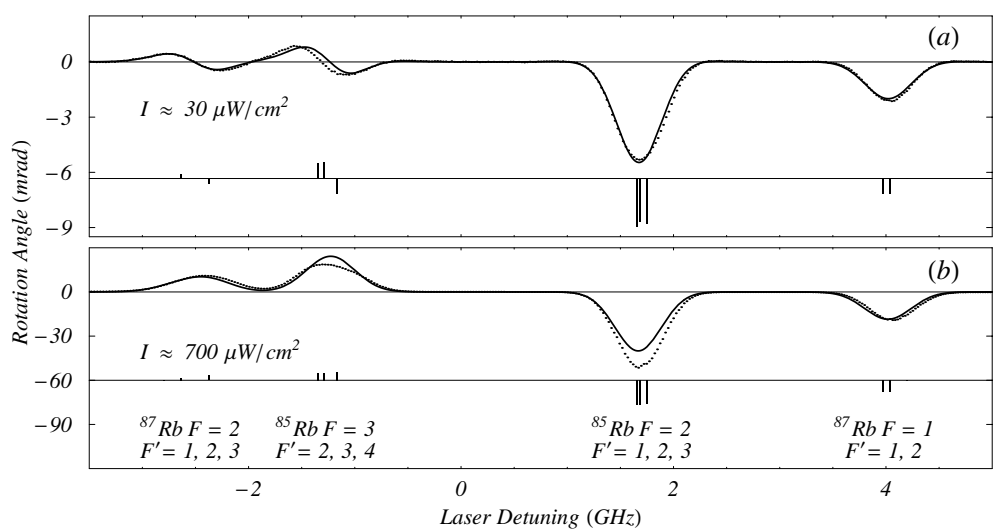

FIG. 2. Comparison of experimental NMOR spectra to density matrix calculations. Dots - data points, solid curves - theory. Offset vertical bars indicate the central frequencies and calculated relative contribution of different hyperfine components $\left(F \rightarrow F^{\prime}\right)$ to the overall rotation. Magnetic field is $\approx 0.1 \mathrm{G}$ (where NMOR is relatively large and coherence effects dominate), laser beam diameter is $\approx 3.5 \mathrm{~mm}$, and $\mathrm{Rb}$ density is $\sim 10^{10}$ atoms $/ \mathrm{cm}^{3}$. The theory assumes an optically thin sample and a uniform spatial light power distribution (scaled to account for absorption). Residual discrepancies between data and experiment are believed to be related to the attenuation of the laser beam as it propagates through the cell.

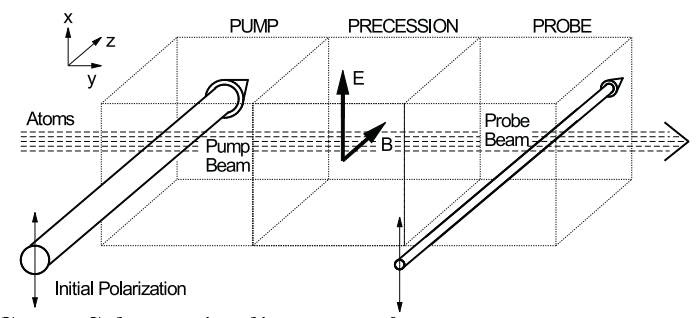

FIG. 3. Schematic diagram of an experiment with separated pump, precession, and probe fields. Atoms move with constant velocity in the $\hat{y}$ direction. The light fields are linearly polarized along the $\hat{x}$ direction.

Figure 4 shows the calculated frequency dependence of NMOR. Also shown are the symmetric and antisymmetric (with respect to probe detuning) contributions to the rotation. The symmetric contribution has a frequency dependence characteristic of the imaginary part of the refractive index and is related to linear dichroism. The antisymmetric contribution, which appears only with nonzero electric field in the precession region, behaves as the real part of the refractive index and is the effect of circular birefringence. This implies that a component of atomic orientation along the $\hat{z}$ direction is created in the precession region.

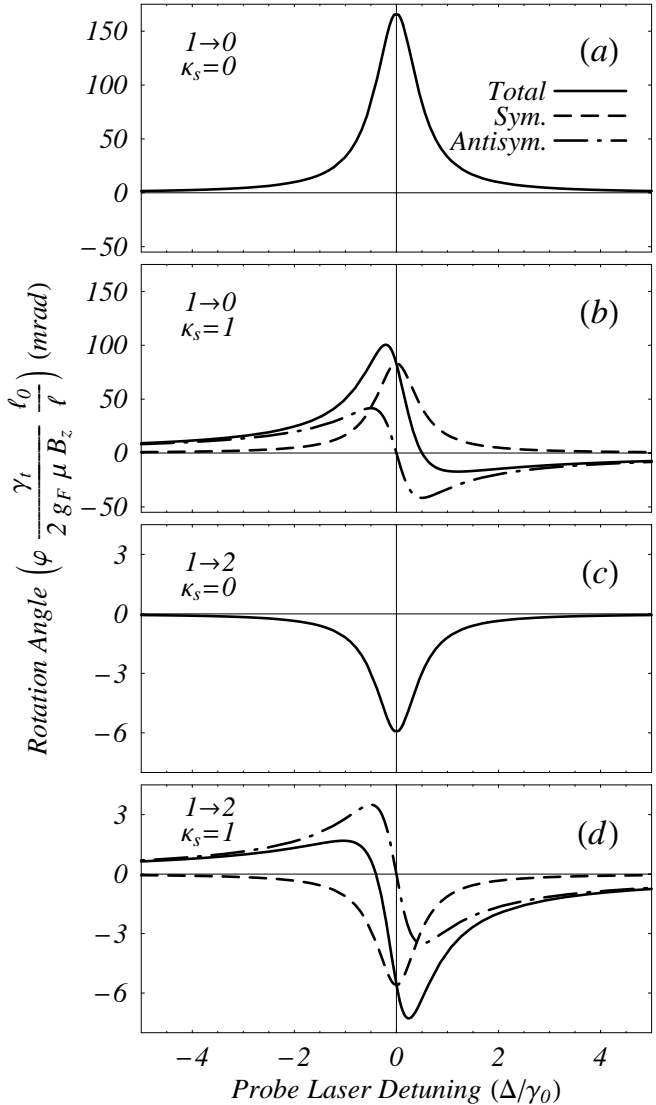

FIG. 4. Calculated Doppler-free optical rotation spectra for separated pump, precession, and probe fields. $\Delta \equiv \omega_{L}-\omega_{0}, \omega_{0}$ is the resonance frequency of the transition and $\omega_{L}$ is the probe light frequency; $l / l_{0}$ is the number of unsaturated absorption lengths; $\kappa_{s}=d^{2} E_{s}^{2} /\left(\omega_{0} \gamma_{t}\right)$. The maximum rotation angle obtained for the $1 \rightarrow 2$ transition is smaller than that for the $1 \rightarrow 0$ case because of less efficient alignment creation via optical pumping and a reduced effect of ground state polarization on absorption and refraction.

The conversion of the atomic alignment produced in the pump region into orientation can be understood in the following way. The alignment (quadrupole moment) produced by optical pumping is along $\hat{x}$. Therefore, as can be seen from symmetry considerations, no orientation can be produced in the precession region by the action of the electric field alone. However, if the axes of the quadrupole moment are rotated with respect to the electric field, alignment can be converted into orientation [8]. The magnetic field in the precession region causes the quadrupole moment $\overleftrightarrow{Q}$ to precess, thus the electric field is able to produce orientation. The induced orientation $\vec{O}$ for small electric $\vec{E}_{s}$ and magnetic $\vec{B}$ fields is given by:

$$
\vec{O} \propto \vec{E}_{s} \times \vec{B} \times\left(\stackrel{\leftrightarrow}{Q} \cdot \vec{E}_{s}\right) .
$$

The sign of the quadrupole moment produced by optical pumping is opposite for $1 \rightarrow 2$ and $1 \rightarrow 0$ transitions, 
therefore $\vec{O}$ is of opposite sign for the two cases. The rotation of the probe light for a given $\vec{O}$ is of opposite sign for $1 \rightarrow 2$ and $1 \rightarrow 0$ transitions (Fig. 5). Thus, for a given probe beam frequency, NMOR due to the induced circular birefringence is of the same overall sign for both transitions.

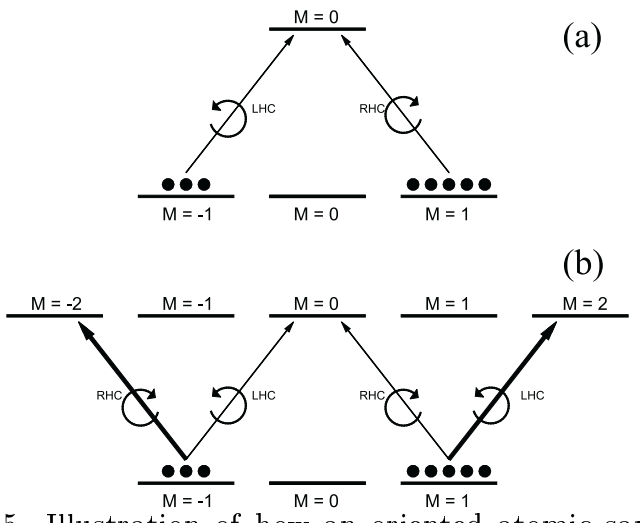

FIG. 5. Illustration of how an oriented atomic sample (a sample with a difference in populations of the $M= \pm 1$ states) causes rotation of opposite signs for $1 \rightarrow 0$ and $1 \rightarrow 2$ transitions. Light with linear polarization is an coherent superposition of left-circularly polarized (LHC) and right-circularly polarized (RHC) light. (a) $1 \rightarrow 0$ case: larger population of the $M=1$ state leads to larger refraction of RHC polarized light, causing counter-clockwise optical rotation (looking into the beam) when light is detuned to the low frequency side of resonance. (b) $1 \rightarrow 2$ case: larger population of the $M=1$ state causes greater refraction of LHC polarized light, leading to clockwise optical rotation for low frequency detuning.

Figure 6 shows the results of density matrix calculations for NMOR with separated regions as a function of the parameter $\kappa_{s}=d^{2} E_{s}^{2} /\left(\omega_{0} \gamma_{t}\right) \ll 1$, which characterizes the Stark shift due to the static electric field. For $\kappa_{s} \ll 1$, NMOR due to circular birefringence is linear in $\kappa_{s}$, as expected from equation (1). Since linear dichroism and circular birefringence produce rotation of opposite signs in the case of the $1 \rightarrow 2$ transition (for the given probe detuning), the overall sign of the NMOR flips when Stark-induced birefringence becomes the dominant cause of the rotation.

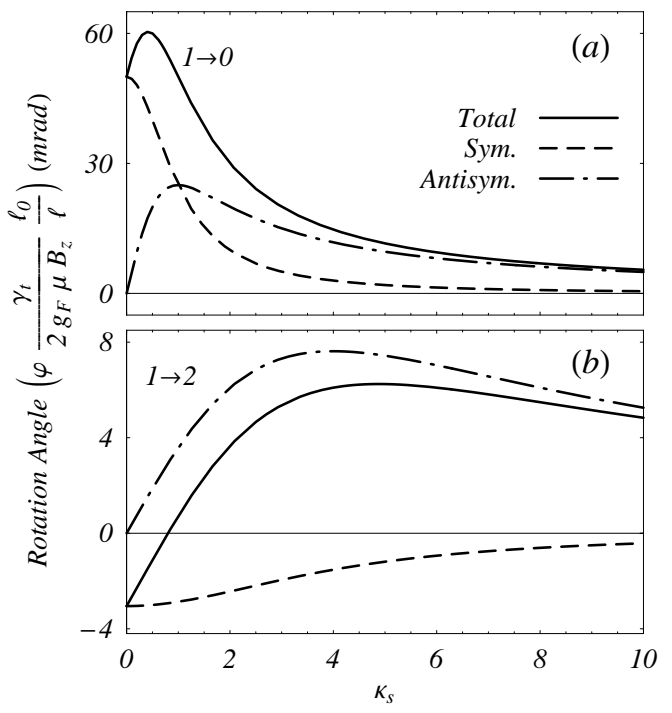

FIG. 6. Calculated Doppler-free optical rotation of probe light as a function of $\kappa_{s}$ for separated pump, precession, and probe fields. Probe light is detuned by $\Delta=-\gamma_{0} / 2$. Note the sign change of the overall rotation for the $1 \rightarrow 2$ case as the electric field is increased.

Now we consider a single region where there is no DC electric field and a single light beam serves as both pump and probe. Here, alignment-to-orientation conversion can occur due to AC Stark shifts produced by the electric field of the light. The AC Stark shifts $\Delta_{A C}$ have a frequency dependence (for the Doppler-free case with $\left.d E_{0}<\gamma_{0}\right)$ given approximately by [15]:

$$
\Delta_{A C} \approx \frac{d^{2} E_{0}^{2}\left(\omega_{L}-\omega_{0}\right)}{4\left(\omega_{L}-\omega_{0}\right)^{2}+\gamma_{0}^{2}}
$$

Figure 7 shows the frequency dependence of NMOR for this case. Note that contribution from AC Stark-induced circular birefringence is symmetric with respect to detuning. This is because NMOR due to circular birefringence is given by a product of two antisymmetric functions. The orientation has an antisymmetric frequency dependence, since it is the product of a Lorentzian function (describing the quadrupole moment created by optical pumping) and the dispersive function from Eq. (2). The rotation angle produced by a given orientation is also dispersive, since it is proportional to the real part of the refractive index. 


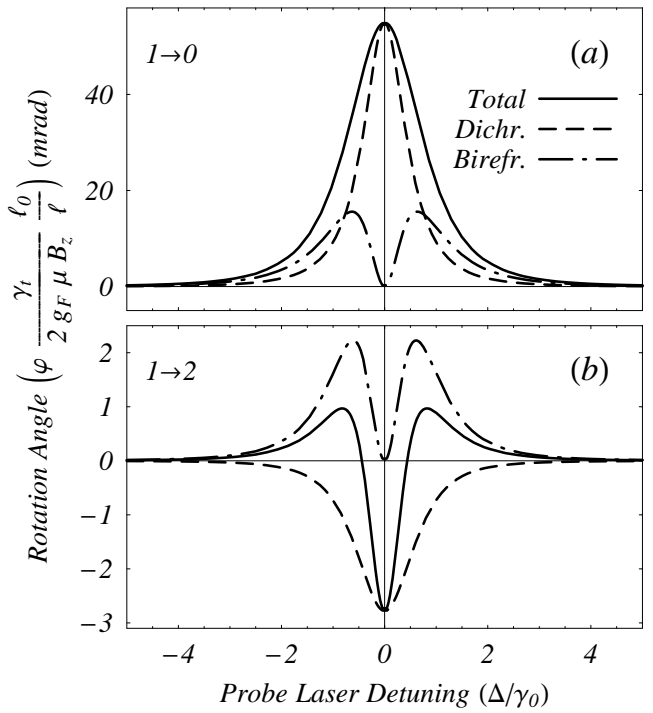

FIG. 7. Calculated Doppler-free optical rotation in an experimental setup similar to that of Fig. 1, with $\kappa=5$. Plots show overall rotation, NMOR due to linear dichroism, and NMOR related to AC Stark-induced circular birefringence.

In the Doppler-free case, optical rotation on resonance is determined by the linear dichroic effect, while both effects contribute off-resonance. In order to describe experimental data, Doppler-free NMOR spectra must be convolved with the atomic velocity distribution. Calculations indicate that in the Doppler-broadened case, NMOR due to circular birefringence dominates at high light powers. This is the regime where NMOR-based magnetometers achieve optimum sensitivity $[4,5]$. For closed $F \rightarrow F+1$ transitions, this effect causes the overall rotation to flip sign, as observed in the experimental data (Fig. 2).

Density matrix calculations also demonstrate that AC Stark shifts play an important role in self-rotation of elliptical polarization [17]. In addition, if an electric field is applied at an angle to the directions of light propagation and linear polarization, Stark shifts can modify optical properties of the medium even in the absence of an applied magnetic field. These effects can be applied in atomic spectroscopy (measurements of electric polarizabilities, etc.) and electromagnetic field measurements [18].

In conclusion, we have considered alignment-toorientation conversion in NMOR, which causes optical rotation via circular birefringence. This effect dominates at high light powers, where $\kappa \gg 1$. It explains a reversal of the sign of rotation for closed $F \rightarrow F+1$ transitions observed in experiments. These results are important for sensitive magnetometry and other applications, including the search for nonlinear optical rotation caused by permanent atomic electric dipole moments $[19,20,4]$.

The authors thank M. Zolotorev and A.I. Okunevich for fruitful discussions. This research is supported by the Office of Naval Research, grant N00014-97-1-0214, and by the U.S. Department of Energy through the
LBNL Nuclear Science Division (Contract No. DEAC03-76SF00098).

* e-mail: budker@socrates.berkeley.edu

[1] W. Gawlik, in Modern Nonlinear Optics edited by M. Evans and S. Kielich, Advances in Chemical Physics Series, Vol. LXXXV, Pt. 3 (Wiley, New York, 1994).

[2] D. Budker, D. J. Orlando, and V. Yashchuk, Am. J. Phys. 67, (1999).

[3] D. Budker, V. Yashchuk, and M. Zolotorev, Phys. Rev. Lett. 81, 5788 (1998).

[4] V. Yashchuk, D. Budker and M. Zolotorev, in Trapped Charged Particles and Fundamental Physics, edited by D.H.E. Dubin and D. Schneider (American Institute of Physics, New York, 1999).

[5] V.A. Sautenkov, M.D. Lukin, C.J. Bednar, G.R. Welch, M. Fleischhauer, V.L. Velichansky, and M.O. Scully, quant-ph/9904032 (1999); M. Fleischhauer, A.B. Matsko, and M.O. Scully, quant-ph/0001072.

[6] S.I. Kanorsky, A. Weis, J. Wurster, and T.W. Hänsch, Phys. Rev. A 47, 1220 (1993).

[7] Here alignment designates the second (quadrupole) polarization moment, and orientation designates the first (dipole) polarization moment, see E. B. Alexandrov, M. P. Chaika, and G. I. Kvostenko, Interference of Atomic States (Springer-Verlag, Berlin, 1993).

[8] M. Lombardi, J. Physique 30, 631 (1969).

[9] C. Cohen-Tannoudji and J. Dupont-Roc, Opt. Commun. 1, 184 (1969).

[10] M. Pinard and C.G. Aminoff, J. Physique 43, 1327 (1982).

[11] R.C. Hilborn, L.R. Hunter, K. Johnson, S.K. Peck, A. Spencer, and J. Watson, Phys. Rev. A 50, 2467 (1994).

[12] R.C. Hilborn, Am. J. Phys. 63, 330 (1995).

[13] This is because for closed transitions, the axis of dichroism corresponding to smaller absorption is initially parallel to the direction of the pump light polarization for $F \rightarrow F-1, F$ transitions (atoms are pumped into a dark state), and perpendicular to the pump light polarization for $F \rightarrow F+1$ transitions (atoms are pumped into a bright state with greater absorption compared to the unpolarized state [A. P. Kazantsev, V. S. Smirnov, A. M. Tumaikin, and I. A. Yagofarov, Opt. Spectrosc. $\mathbf{5 7}(2), 116$ (1984)]).

[14] Our calculations are based on principles similar to those applied in F. Schuller, M.J.D. Macpherson, and D.N. Stacey, Physica C 147, 321 (1987); F. Schuller, M.J.D. Macpherson, and D.N. Stacey, Opt. Commun. 71, 61 (1989); M.G. Kozlov, Opt. Spectrosc. (USSR) 67, 789 (1989); S.V. Fomichev, J. Phys. B 24, 4695 (1991); K.P. Zetie, R.B. Warrington, M.J.D. Macpherson, D.N. Stacey, and F. Schuller, Opt. Commun. 91, 210 (1992); F. Schuller, D.N. Stacey, R.B. Warrington, and K.P. Zetie, J. Phys. B 28, 3783 (1995); G. Nienhuis and F. Schuller, Opt. Commun. 151, 40 (1998). 
[15] Under the stated conditions, this formula is a good approximation of the expression obtained from dressed state considerations for a two-level atom in, e.g., C. Cohen-Tannoudji, Metrologia 13, 161 (1977).

[16] E.B. Alexandrov, M.V. Balabas, A.S. Pasgalev, A.K. Vershovskii, and N.N. Yakobson, Laser Phys. 6, 244 (1996).

[17] D. Budker, R. Y. Chiao, D. S. Hsiung, S. M. Rochester, and V. V. Yashchuk, Technical Digest of Conference on Lasers and Electro-Optics, Quantum Electronics and Laser Science, (2000).

[18] D. Budker, D.F. Kimball, S.M. Rochester, and V.V. Yashchuk, Report LBNL PUB-5453, (1999).

[19] L. M. Barkov, M. S. Zolotorev and D. Melik-Pashayev, Sov. JETP Pis'ma 48, 144 (1988); L.M. Barkov, D. A. Melik-Pashayev, and M.S. Zolotorev, Opt. Commun. 70, 467 (1989).

[20] B. Schuh, S.I. Kanorsky, A. Weis and T.W. Hänsch, Opt. Commun. 100, 451 (1993). 